\title{
Sero-prevalence of Transfusion Transmissible Infections Markers Among Blood Donors Attending the Blood Transfusion Department Of A Teaching Hospital in Chittagong
}

\begin{abstract}
As per Safe Blood Transfusion Law 2002 of Bangladesh and worldwide strategy of screening of Transfusion Transmissible Infections (TTIS) markers in blood donors of various categories is a must for the prevention of Human Immunodeficiency Virus/Acquired Immunodeficiency Syndrome (HIV/AIDS), Hepatitis $B$ virus $(H B V)$, Hepatitis $C$ virus (HCV), Syphilis and Malaria with a aim to decrease morbidity and mortality of the patients receiving blood and blood components. A total of 33,553 blood donors attending the Blood Transfusion Department of Chittagong Medical College Hospital from January 2009 to December 2009 amongst which replacement or relative donors were 30868 (92\%), voluntary donors 2685 (8\%) and male donors 27722 (82.62\%), female donors 5831 (17.38\%). Among the donors 15133 (45.10\%), were of age group between 18-25 years, 17809 (53.08\%) were of age group between 26-45 years, and 611 (1.82\%) were of age group between 46-60 years. The purpose of this study was to determine the sero-prevalence
\end{abstract}

Keywords: Safe blood, Blood transfusion, HIV/AIDS, HBV, HCV, Syphilis, Malaria.

\section{Introduction}

According to World Health Organization (WHO) study and data around the world regarding blood safety, about $80 \%$ of world's population does not have access to safe blood (Global Data Base). Despite limited resources, rapid spread of HIV/AIDS and other TTIs, transfusion of safe blood and blood component has to be ensured to reduce morbidity and mortality. ${ }^{1}$ Screening of blood and blood components varies from country to country depending on the availability of resources and adequate trained manpower. The objective of this study was to determine the sero-prevalence of HIV, HBV, HCV, Syphilis and Malaria in blood donors attending in Blood Transfusion Department of Chittagong Medical College Hospital, Chittagong, Bangladesh. Since the inception of Safe Blood Transfusion program, screening of the donated blood for TTIs has emerged a new era in Bangladesh. As per Safe Blood Transfusion Law-2002, screening of HBV, HCV, HIV, Syphilis and Malaria is mandatory. Since the emergence of HIV/AIDS, it has been the greatest health hazards ever faced by mankind. HIV/AIDS epidemic has already killed more than 20

1.Dr. Rehana Aziz, Assistant Professor of Biochemistry, Chittagong Medical College, Chittagong.

2. Dr. Shameem Hyder, Professor of Blood Transfusion, Chittagong Medical College \& Hospital, Chittagong.

3. Dr. Ajoy Deb, Lecturer of Biochemistry, Chittagong Medical College, Chittagong.

4.Dr. Mahmudul Haque, Professor of Biochemistry, Chittagong Medical College, Chittagong.

5. Dr. A Q M Serajul Islam, Professor of Dermatology \& STDs, Chittagong Medical College \& Hospital, Chittagong.

Corresponding Author

Dr. Rehana Aziz Assistant Professor of Biochemistry, Chittagong Medical College, Chittagong. of HIV, HBV, HCV, Syphilis and Malaria in blood donors in a Teaching Hospital at Chittagong, Bangladesh.

Blood samples from selected donors were tested for HIV, HBV $H C V$ and Malaria by Immunochromatographic (ICT) rapid test device, Syphilis by rapid plasma reagin (RPR) test device method.

Screening procedures were in accordance with the manufacturer's instruction in terms of adding serum, adding buffer and time duration of reading results. Screening result after compilation shows HIV was reactive in 7 (0.02\%) donors all of which were male. HBV was reactive in 285 (0.849\%) donors, out of which 272 (95.43\%) were male and 13 (4.57\%) were female. $\mathrm{HCV}$ was reactive in $23(0.068 \%)$ donors, out of which 18 (78.24\%) was male and 5 (21.76\%) were female. Syphilis was reactive in 32 (0.095\%) donors, out of which 29 $(90.62 \%)$ was male and $3(9.38 \%)$ were female. Malaria was reactive in $31(0.09 \%)$ donors all of which were male. By studing this prevalence, attention will be drawn towards severity of the situation so that effective measure can be taken to prevent further transmission of TTIs by blood transfusion.

million people globally. At present more than 40 million people living with HIV/AIDS all over the world. In South East Asian countries more than 6.7 million people living with HIV/AIDS. About 3 million peoples are dying of HIV/AIDS every year. ${ }^{2}$ To determine the prevalence of $\mathrm{HBV}$ and $\mathrm{HCV}$, comprehensive study has yet to be done. However, different studies have shown that there is significant number of HBV and HCV carriers in Bangladesh. Due to emergence of HBV mutants, for blood safety, their implications have become a major concern for research. One study of 576 apparently healthy male adults revealed that $7.8 \%$ were HBV carriers and about $60 \%$ of post transfusion hepatitis due to $\mathrm{HBV}^{3}$. Another study conducted among 163 professional donors and 83 voluntary donors revealed that $1.2 \%$ professional donors were sero-positive for $\mathrm{HCV}$ and $29 \%$ of the voluntary donors were $\mathrm{HBsAg}$ positive. ${ }^{4}$

This study may be useful in formulating transfusion therapy and also in developing prevention policy for safe blood.

\section{Materials and Methods}

A cross sectional descriptive study was carried out in the Blood Transfusion Department of Chittagong Medical College Hospital $(\mathrm{CMCH})$ during the period of January 2009 to December 2009 to determine the seropositivity of TTIs markers in blood donors. Data of routine screening reports of blood donors were collected from blood bank coming to blood transfusion department of this institution. A total of 33,553 blood donors attending the blood bank 
amongst which male donors 27722 (82.62\%), female donors $5831(17.38 \%)$. Subject of age group between 18-60 years were included in this study. No professional blood donors were selected in this study. According to WHO guideline the current strategy for the detection of infection markers in blood donor is to reject the reactive one in a single test and that has been followed in this centre. Blood samples from selected donors were tested for HIV, HBV HCV and Malaria by Immunochromatographic (ICT) rapid test device, Syphilis by rapid plasma reagin (RPR) test device method. Chi square test was done to analyze and interpret data. The findings of report after compilation were summarized in various tables and descriptic statistics were determined in term of percentage.

\section{Results}

A total of 33,553 blood donors either replacement and / or voluntary donors were screened for seropositivity of $\mathrm{HBV}$, $\mathrm{HCV}$, Malaria, Syphilis and HIV according to WHO set criteria. Among the donors, 378 (1.12\%) had TTIs. Hepatitis B was present in 285(0.84\%), 23(0.068\%) were positive for Hepatitis $\mathrm{C}$ virus, 32(0.095) were positive for Syphilis, 31(0.09\%) were positive for Malaria and 7 $(0.02 \%)$ were positive for HIV.

Table I: Gender distribution of blood donors at CMCH in the year 2009.

\begin{tabular}{lll|}
\hline Blood donors & $\mathrm{n}=33553$ & percentage \\
Male & 27722 & $82.62 \%$ \\
Female & 5831 & $17.38 \%$ \\
\hline
\end{tabular}

This table shows that the percentage of male blood donors were more than 4 times higher than female blood donors.

Table II: Age distribution of blood donors at $\mathrm{CMCH}$ in the year 2009.

\begin{tabular}{|lcc|}
\hline Age of blood donors in & Total numbers & percentage \\
years & & \\
$18-25$ & 15133 & $45.10 \%$ \\
$26-45$ & 17809 & $53.08 \%$ \\
$56-60$ & 611 & $1.82 \%$ \\
\hline
\end{tabular}

Age group of 26-45 years blood donors were higher incidence of blood donations.
Table III: Sero-prevalence of TTIs markers in blood donors at CMCH in the year 2009.

\begin{tabular}{|c|c|c|c|}
\hline \multirow[t]{8}{*}{ Total blood donors } & TTIs Markers & \multicolumn{2}{|c|}{$\begin{array}{l}\text { No. oferos - Percentage } \\
\text { positive blood }\end{array}$} \\
\hline & $\mathrm{HbsAg}$ & 285 & $0.84 \%$ \\
\hline & Anti-HCV & 23 & $0.068 \%$ \\
\hline & M.P & 31 & $0.09 \%$ \\
\hline & RPR & 32 & $0.095 \%$ \\
\hline & AntHIV & 7 & $0.02 \%$ \\
\hline & Total & 378 of & $1.12 \%$ \\
\hline & seropositive don & & \\
\hline
\end{tabular}

Results of this study shown that $1.12 \%$ of total blood donors have TTIs, prevalence of HBV infection was higher than other TTIs markers.

Table IV: Distribution of sero-positivity of TTIs markers among blood donors in terms of sex.

\begin{tabular}{|c|c|c|c|c|}
\hline TTIs markers & $\begin{array}{l}\text { Total sero } \\
\text { donors }\end{array}$ & positive blood & $\begin{array}{l}\text { Male } \\
\text { blood donors }\end{array}$ & $\begin{array}{l}\text { Female blood } \\
\text { donors }\end{array}$ \\
\hline HBsAg & 285 & & $272(95.43 \%)$ & $13(4.57 \%)$ \\
\hline Anti-HCV & 23 & & $18(78.26 \%)$ & $05(21.73 \%)$ \\
\hline Anti - HIV & 7 & & $07(100 \%)$ & Nil \\
\hline RPR & 32 & & $29(90.62 \%)$ & $03(9.38 \%)$ \\
\hline M.P & 31 & & $100 \%)$ & Nil \\
\hline
\end{tabular}

Prevalence of HBV among male donors (95.43\%) was statistically significant $(\mathrm{p}>0.05)$ compared to $\mathrm{HBV}$ prevalence among females donors $(4.57 \%)$. HCV and Syphilis prevalence were high among male blood donors (78.26\%) and $(90.62 \%)$ respectively, these were not significant $(\mathrm{p}<0.05)$ compared to HCV and Syphilis prevalence among females blood donors. There was no HIV and Malaria infections were found in female blood donors. It may be due to unexplianed confounding variables in the study cases. The study was based only on records of a blood bank and it may be proposed to multi centric and generalized study all over the country with a bigger sample size.

\section{Discussion}

Interesting and encouraging findings have been revealed out of this study. In this study there is male preponderence of TTIs markers. HIV was reactive in $7(0.02 \%)$ donors all of which were male. HBV was reactive in $285(0.849 \%)$ donors, out of which $272(95.43 \%)$ were male and 13 
(4.57\%) were female. HCV was reactive in $23(0.068 \%)$ donors, out of which $18(78.24 \%)$ was male and 5 $(21.76 \%)$ were female. Syphilis was reactive in 32 $(0.095 \%)$ donors, out of which $29(90.62 \%)$ was male and $3(9.38 \%)$ were female. Malaria was reactive in $31(0.09 \%)$ donors all of which were male. The prevalence of HIV, HBV, and HCV and Syphilis positivity in Indian blood donors is HIV- $0.084 \%$ to $3.87 \%$, HBV - $0.66 \%$ to $12 \%, \mathrm{HCV}-0.5 \%$ to $1.5 \%$ and Syphilis $-0.85 \%$ to $3 \%$ respectively. ${ }^{5}$ The present study revealed seroprevalence of HIV $0.02 \%$ in the donors lower as reported by other studies. ${ }^{8,6}$ The seroprevalence of $\mathrm{HBV}$ was $0.84 \%$ similar to that reported by the other studies $0.99 \% 8$ and $0.66 \%$, whereas variable results of $2.45 \%,{ }^{8} 5.86 \%, 25 \%{ }^{9}$ have also been reported. The seroprevalence of $\mathrm{HCV}$ was $0.068 \%$ which is much lower as reported by other studies $0.19 \% 8,0.88 \%,{ }^{10} 1.09 \%{ }^{11}$ and $6.21 \%{ }^{12}$ The seroprevalence of Syphilis was $0.095 \%$ in the present study which is lower than other studies. ${ }^{13}$ The decrease in seroprevalence of TTIs markers may have been mainly associated with improved donor selection practices according to definite criteria and exclusion of repeat donors screened reactive on previous donation. In a study it has been estimated that the risk was 1 in 89,000 donations collected in the window period of organism. History of blood donors during interview, discussion and awareness, motivation on blood safety among blood donors has considerable impact on reduction of TTIs markers and thereby reducing morbidity and mortality.

\section{Conclusion:}

The study does not reflect the actual sero-prevalence of TTIs markers among the blood donors of the country. To improve the effectiveness of screening up to desirable level there should be detection of post-transfusion TTIs markers. The evaluation of testing techniques is to be done from time to time and their regular monitoring by periodic External Quality Assurance System (EQAS). Recruitment of low risk voluntary blood donors should be strictly maintained. Every reporting should be done by improved system available at the centre concerned. Overall, the findings of the study are an encouraging one and support the decrease in trends of sero-prevalence of TTIs markers in this region of the country. It is important to follow the WHO guidelines to screen every donor for HBV, HCV, HIV, Syphilis and Malaria to decrease the transmission of TTIs. Record keeping should be promoted to follow up the screened patients. However, extensive and multi center studies are needed to find out the prevalence of two or three TTIs markers in the same individuals.

\section{References}

1. Screen all donated blood for infectious agent. Health Topic:WHO/BCT/02.02;1.

2. Guidelines for HIV diagnosis and monitoring of antiretroviral therapy.SEA-HLM-382 (Rev.1): WHO Project: ICPBCT001.December 2005;7.

3. Bangladesh Medical Research Council (BMRC) Bulletin, 1984:10(1);1-6.

4. Khan M, Husain M, Yang M, Hashzuma K, Yousuf M, Tanaka E, Matsumoto A, Future S, Kiyosawa K. Comparison of seroepidemiology of hepatitis $\mathrm{C}$ in blood donors of Bangladesh and Japan. Gastroenterology Japonica. 1993:28 (Supp 5):28-61.

5. Garg S, Mathur DR, Garg DK. Comparison of seropositivity of HIV, HBV, HCV and Syphilis in replacement and voluntary blood donors in western India. Indian J Pathol Microbiol 2001;44:409-12.

6. Telatela SP, Matee MI, Munubhi EK. Seroprevalence of hepatitis B and C viral co- Infections among children infected with human immunodeficiency virus attending the paediatric HIV care and treatment center at Muhimbili National Hospital in Dar-es- Salaam, Tanzania. BMC Public Health 2007; 7:338.

7. Singh B, Verma M, Kotru M, Verma K, Batra M. Prevalence of HIV and VDRL seropositivity in blood donors of Delhi. Indian J Med Res 2005; 122:234-6.

8. Nanu A, Sharma SP, Chatterjee K, Jyoti P. Markers for transfusion-transmissible Infections in north Indian voluntary and replacement blood donors: Prevalence and Trends 19891996. Vox Sang 1997; 73:70-3.

9 .Singh B, Kataria SP, Gupta R. Infectious markers in blood donors of East Delhi: Prevalence and trends. Indian J Pathol Microbiol 2004; 47:477-9.

10. Sharma RR, Cheema R, Vajpayee M, Rao U, Kumar S, Marwaha N, et al. Prevalence of markers of transfusion transmissible diseases in voluntary and replacement blood Donors. Natl Med J India 2004; 17:19-21.

11. Nanda A, Tyagi S, Basu S, Marwaha N. Prevalence of transfusion transmitted Infections among voluntary and replacement donors. Indian J Hemat Blood Transf 2001; 19:104-5.

12. Jain M, Chakravarti A, Verma V, Bhalla P. Seroprevalence of hepatitis viruses in Patients infected with the human immunodeficiency virus. Indian J Pathol Microbiol 2009; 52:17-19.

13. Zhang J, Zon S, Ginlivi A. Epidemiology of hepatitis B in Canada. Can J. Infect. Dis. 2001:12; 345-350. 